\title{
Can a well balanced soft tissue envelope speed up the postoperative treatment and improve clinical follow-up? A control - pilot study using a dynamic ligament balancing device based on functional stability
}

\author{
Thomas Paszicsnyek ${ }^{1}$, Edoardo Bori ${ }^{2}$, Christoph Stiegler ${ }^{1}$, Bernardo Innocenti ${ }^{2}$ \\ ${ }^{1}$ Privatklinik Graz Ragnitz, Austria \\ ${ }^{2}$ BEAMS Universite de Bruxelles \\ tp@ortho-unfall.at, edoardo.bori@gmail.com, bernardo.innocenti@ulb.ac.be
}

\section{Introduction}

Worldwide, the number of TKA implants is increasing. The reasons are higher demands from elder patients, who are limited by joint degeneration and pain and the expectations of a better quality of life. Even if registry demonstrate that TKA has high satisfaction rate, there are still between $15 \%$ and $20 \%$ dissatisfied patients. In some cases, it's hard to find the proper reason, but it is reasonable to suppose that most of these patients are suffering from a mismatch in the ligament balance and bony symmetry of the artificial joint.

\section{Materials and Methods}

The proper soft tissue balancing is one of the most discussed topics of the last years. We initiated a study using an electronic device ("Dynamic Ligament Balancing sensorplate", or "DLB") to compare the benefit of the measurement of joint pressure, space and position in comparison to a conventional surgical procedure. Besides that, we followed the concept of functional stability, which tells us that a tension of $40 \mathrm{~N}$ in total is sufficient to reach proper (functional) joint stability.

This control pilot study was set up as a single surgeon, single center study and consists of 25 patients treated by the use of the sensorplate and a control group of 25 patients, treated in a conventional setup. 
We used the following scores for evaluation: OKS, AKSS and FJS, preoperatively and during the FU examinations (postoperative, 6 weeks, 3 months, 6 months and 1 year), the FJS was only used preoperatively, after 6 months and 1 year FU.

Beside scoring, clinical examination and routine x-ray we performed an EMG testing at all FU dates. The EMG testing was set up under a dynamic protocol during walking on a treadmill, stepping up and down and contraction of the muscle against a defined resistance. For the EMG testing we used a Noraxon wireless scientific EMG device and a Frei Factum Novum knee flexion/extension unit.

\section{Results}

The study was performed between January 2017 and May 2019. The mean age of the patients was in average 72 years, $66 \%$ female and $34 \%$ male. After 1 year, results demonstrate a clear difference in the development of the postoperative situation between dynamic balanced TKA and the control one. All patients passed the fu examinations and the following results could be highlighted:

- the OKS in DLB group pass from an average of 28.5 preoperatively to 44,1 at last FU;

- in the control group the OK pass from an average of 16,5 to 40,32 at last FU;

- the AKSS KS/FS in DLB group pass from an average of 32.29/38.1 to 91.1/97 at last FU;

- In the control group AKSS KS/FS KS pass from an average of 40,25/FS 53,75 to 91,1/93,64;

- In the DLB group the FJS improve from average 17.06 preoperatively to 67,3 at last FU;

- In the control group the FJS improve from average 32.50 preoperatively to 75.93 at last $\mathrm{FU}$;

The use of such electronic device then showed to be improving the soft tissue envelope stability, enabling a significantly better patient FU, especially in terms of OKS

\section{Discussion}

The DLB system is a new option to value and improve the soft tissue envelope tensioning during the surgical TKA operation. It allows to measure contact pressure, slope and joint space all over the entire ROM.

This is the first time to evaluate the midflex situation during the surgery and to avoid midflex instability and contracture, even asymmetry and mismatch in flexion and extension. Knowledge about the geometry of the prosthesis (single radius, j-curve, symmetry...) is mandatory to make the right interpretation of the achieved results. Also there is the verification that less tension than the one usually applied during surgeries is sufficient to stabilize the knee. 


\section{Conclusion}

Using an electronic device for measurement is an advanced option to improve patient satisfaction after total knee arthroplasty. Like the studies of other existing devices have shown before there is a massive change in the kinematic behavior of the muscular abilities by using these tools for a better soft tissue balance. The DLB system is another option by showing 3 different measurement results (pressure, distance and joint angle) to adapt the implantation procedure to the individual situation of the patient. It is a time efficient tool without an extension of the surgical time, simple to handle and easy to use.

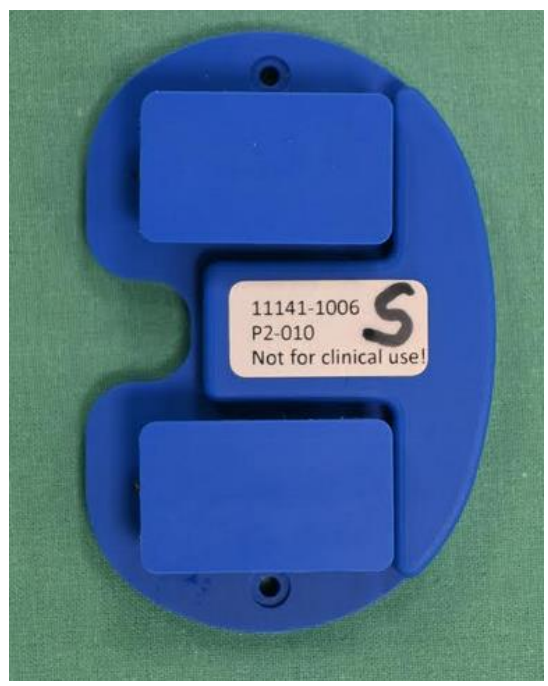

Figure 1: The DLB device (from above)

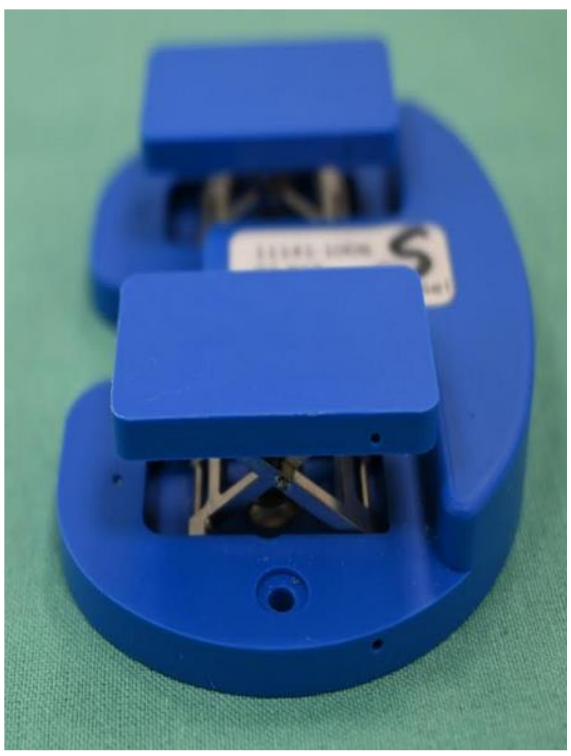

Figure 2: The DLB device (lateral view) 